Revista Calidad en la Educación Superior

Programa de Autoevaluación Académica

Universidad Estatal a Distancia, Costa Rica

ISSN 1659-4703

revistacalidad@uned.ac.cr

\title{
LA VIRTUALIDAD EN LOS PROCESOS FORMATIVOS ACTUALES
}

\author{
VIRTUALITY ON CURRENT FORMATIVE PROCESSES
}

\section{Arturo Azofeifa-Céspedes ${ }^{1}$ aazofeifa@uned.ac.cr Universidad Estatal a Distancia, Costa Rica}

\author{
Volumen 7, Número 2 \\ Noviembre, 2016 \\ pp. $129-142$
}

Recibido: 22 de setiembre, 2016

Aprobado: 30 de octubre, 2016

\footnotetext{
${ }^{1}$ Magister en Administración de Proyectos. Magister en Auditoría Financiera Forense. Actualmente es Investigador en el Centro de Investigación y Evaluación Institucional de la UNED. Profesor de la cátedra de Ciencias Policiales de la UNED. Profesor universitario en diferentes asignaturas, grados, carreras y universidades. Correos electrónicos: aazofeifa@uned.ac.cr / arturoazofeifa@gmail.com
} 


\section{Resumen}

Los procesos formativos deben caracterizarse por la actualización constante que pueda responder a las necesidades de los contextos en los que se desarrollan. Es así como la tecnología ha irrumpido de tal manera que pensar en educación sin virtualidad, es cada vez menos probable, y sus aplicaciones se extienden no sólo a los contextos formativos tradicionales (escuela, colegio y universidad), sino a los contextos laborales. Estos nuevos modelos de aprendizaje suponen muchas ventajas que incluyen ahorro de recursos, posibilidad de realizar formación y capacitación a la distancia, entre otros, y al mismo tiempo plantean serios cuestionamientos sobre lo que se entiende por procesos formativos y sus actores, como el papel de profesor-alumno. Es innegable que la tecnología estará cada vez más implicada en los procesos formativos virtuales, por lo que estas preguntas y valoraciones deberán replantearse y discutirse.

Palabras clave: Virtualidad, procesos formativos, educación, instituciones educativas, interacción profesor-alumno, tecnología, capacitación.

\section{Abstract}

The training processes should characterize is by the update constant that can respond to the needs of them contexts in which is developed. It is as well as technology has burst so that thinking about virtual education is less and less likely, its applications extend not only to the traditional educational contexts (school, College and University), but to those contexts labour. These new models of learning are many advantages including saving resources, possibility to carry out education and training to the distance among others, while we face serious questions about what we understand by training processes and their actors, as the role of teacher-student. Is undeniable that the technology will be each time more embedded in them processes training virtual, by what these questions and assessments must rethink is and discuss is.

Keywords: Virtuality, training processes, education, educational institutions, interaction teacher-student, technology, training.

\section{Introducción}

Si los tiempos actuales se caracterizan es por la innovación, la rapidez y la constante inserción de la tecnología en todos los campos del conocimiento humano. Al avocarse en este ensayo sobre el papel de la virtualidad en los procesos formativos actuales, es necesario mencionar como la inserción de la tecnología en estos procesos de 
transmisión del conocimiento ha revolucionado la manera como se aprende y se trabaja.

Es válido entonces remontarse a como históricamente los seres humanos han transmitido el conocimiento de generación en generación. En tiempos ancestrales -y al no poseer otros medios- era la transmisión oral la que se encargaba de preservar las experiencias, costumbres, tradiciones, mitos, y en general todo el conocimiento que se poseía. Luego, con la invención de la escritura, esta empezó a tomar un rol fundamental que se extiende hasta la actualidad (es factible tener medios electrónicos pero al fin y al cabo los mismos son "escritos"); y de los que difícilmente se pueda llegar a prescindir.

Asimismo, las primeras escuelas, institutos de formación técnica y en especial las primeras universidades se encargaron de dar forma a los modelos de enseñanza tradicional que se conoce y que prevalecieron hasta la actualidad. El elemento que tendrían en común todas ellas sería los profesores, que son aquellas personas que por su formación académica y experiencia de vida (no se puede negar que es un conjunto de ambas), son los llamados precisamente a hacer la transmisión de conocimiento a sus alumnos. Por supuesto, dependiendo del área del saber la que se hable se requiere más formación a nivel teórico o práctico, pero el rol del profesor como facilitador es innegable y se ha mantenido invariablemente definido a través de cientos de años y hasta nuestros tiempos e instituciones actuales.

La revolución tecnológica, empeñada en trastocar todas las áreas de la experiencia humana ha empezado a redefinir la manera en cómo se llevan a cabo los procesos formativos actuales. La irrupción de la computadora primero, y del internet después, supuso un cambio que aún no se ha terminado de asimilar, pero que indudablemente, como toda revolución, vino a cuestionarnos nuestra manera de hacer las cosas. Hoy por hoy, difícilmente, existen casas de enseñanza que no integren la virtualidad en sus 
currículos, y más bien la constante renovación de los mismos se ve como una necesidad apremiante.

\section{La creciente importancia de la innovación tecnológica}

Como señalan (Barberà Gregori \& Badia Garganté, 2005, p. 2), el desarrollo de nuevas modalidades de enseñanza a "distancia tecnológica" pone cada vez más en duda la diferenciación tradicional existente entre educación presencial y educación a distancia. Pero no sólo eso, sino que han llevado ante la necesidad de replantear los procesos mismos de enseñanza presencial que se llevan a cabo en las aulas de las instituciones educativas, especialmente cuando existe un fuerte movimiento que propone combinar el aula presencial con el aula virtual (conocido como "blended learning").

Algunas de las ventajas de integrar la virtualidad en los procesos formativos saltan a la vista. Por ejemplo, un ejecutivo que trabaje en una transnacional en Costa Rica podría formarse en tiempo real con su par en Europa o en China. Hasta hace muy poco eso era algo totalmente impensado, y de haberse hecho tendría que haber sido por otros medios como el teléfono, que claramente supone una serie de desventajas comparativas con una videoconferencia, por ejemplo. De otras maneras había pocas opciones de formarse en tiempo real.

En este mismo ejemplo, esos ejecutivos podrían estar desempeñando tareas necesarias para ese proceso formativo simultáneamente y desde sus computadoras. Así, si fueran desarrolladores de software por ejemplo, un ejecutivo podría estar recibiendo clases sobre cómo programar en cierto lenguaje específico, y a la vez escribir un código de prueba conforme avanza la clase. Podrían igualmente estar compartiendo contenidos, direcciones, datos clave o bien simplemente apuntes.

Si ese mismo proceso tuviera que hacerse sin utilizar medios virtuales, entonces se tendría que hablar de una gran inversión en términos monetarios, pues uno de las dos 
partes debería desplazarse hasta donde está la otra. O bien, se podría hablar de medios alternativos que se podrían utilizar, como el correo, pero este implicaría inconvenientes obvios en términos no sólo de tiempo, sino de imposibilidad para establecer esa supervisión y feedback en tiempo real del cual se habló anteriormente.

Como una manera de enfocarse acerca de lo que representa la virtualidad en procesos formativos dirigidos especialmente en el plano laboral, es posible señalar que ha llegado a convertirse en una poderosa herramienta con la que debe contar toda empresa, ya que esta no solamente se utiliza para agilizar los procesos operativos y administrativos, presentar sus datos, reportes o información, sino que también esta ejerce múltiples funciones. Esto aunado a que una empresa se tiene que ir adaptando a los cambios que se presentan en el mercado, entre ellos los cambios tecnológicos.

Entre esas múltiples funciones, se enfatiza en el rol de formador de los otros colaboradores de una empresa. Como es de conocimiento de todos, es necesario que los trabajadores aumenten no solo su conocimiento en el área en que se desempeñan, sino que este se extienda a otras áreas, es decir, que aprendan a realizar otras laborales o bien actualicen el conocimiento con que ya cuentan.

Como bien lo señala (Calvo Verdú, 2005), al incorporar la virtualidad en los procesos de formación o capacitación, se despliega una serie beneficios tanto para el trabajador que recibe la capacitación como también para la organización, entre estas ventajas se contemplan las siguientes:

Sobre sus beneficios económicos, estos se expresan en la reducción de una serie de costos, por ejemplo: los gastos de traslados de personal, si es el caso que se requiera que el trabajador se desplace a otro lugar a recibir la capacitación, este traslado conlleva a que el mismo se le tenga que pagar viáticos, esto significa que, además del pago por concepto de transporte, se genera un gasto adicional por alimentación. Todos estos costos sumados a otras eventualidades tales como al 
hecho de que la empresa requiera contratar el instructor, sea este interno o externo, disponer del espacio adecuado o disponible para impartir sus capacitaciones, la impresión de materiales, entre otros.

Asimismo, esto implica también que los trabajadores eviten trasladarse desde su casa u oficina hacia otro lugar, ya que en muchas ocasiones se dan capacitaciones cuya duración es de varios días, por lo cual se ven en la obligación de dejar a sus familias durante este período, generando un inconveniente.

> En cuanto a los beneficios para el trabajador, estos se traducen en primera instancia a que toda formación le brinda un plus al empleado que la recibe, independientemente como la reciba, es decir, si esta es presencial o virtual.

En segunda instancia, otro de los grandes beneficios de contar con esta herramienta es que a través de la virtualidad una gran cantidad de personal se puede capacitar al mismo tiempo, independientemente del lugar o país en que estén, un caso de estos son las llamadas videoconferencias, las cuales son vistas por varias personas y transmitidas a diferentes lugares. Además de esto, estas tienen la ventaja de que se pueden grabar, lo que favorece que los participantes pueden volver a escuchar la información dada, o bien aquellos que no pudieron asistir puedan ingresar a su computadora y obtener esa información. Además de las videoconferencias, se cuenta con sistemas como skype, en el cual permiten la interacción de personas. Conforme transcurre el tiempo, los avances tecnológicos son mayores y se desarrollan otros sistemas más ágiles y funcionales que permiten o facilitan la formación de trabajadores online, es decir, a través de internet.

También, gracias a la virtualidad, ahora es posible tener a disposición una gran cantidad de recursos que de otra manera sería difícil sino imposible. Un buen ejemplo de ello son las bibliotecas virtuales mediante las cuales obtenemos libros y materiales de apoyo desde cualquier parte del mundo, y sobre cualquier tema que se necesite desarrollar. Así, en un sol flash drive es posible tener disponible gran cantidad de libros al alcance de la mano, lo cual a todas luces podría facilitar grandemente los procesos de investigación con en el ámbito educativo y laboral. 
Sin embargo, este cambio que supone la irrupción de la virtualidad en los procesos formativos, también genera una serie de cuestionamientos al respecto, sobre su conveniencia y la mejor manera de insertarlo en los procesos formativos tradicionales. Tal vez uno de los primeros puntos es el nuevo rol que deben asumir los profesores ante la necesidad de incluir la los dispositivos tecnológicos en las clases. Es decir, si la como institución educativa tiene la posibilidad de impartir contenidos a través de medios virtuales, debería plantearse la pregunta: ¿siguen siendo necesarios los profesores?

Hasta ahora la postura más generalizada es que sí. Es decir, se toma como base que el o la profesora sean los encargados de impartir los contenidos de los cursos y que los medios virtuales sean sólo un complemento del cual echar manos para sumar recursos, promover actividades, dejar tareas, etcétera. Así, muchos de los trabajos deben ser entregados por estos medios lo cual supone un nuevo dialogo profesor-alumno que se rige por las mismas reglas de la virtualidad y que no se había visto antes.

Ya los plazos de entrega, por ejemplo, no se limitan a las horas clase, sino que se puede jugar con esos elementos para flexibilizar y favorecer estos canales de comunicación. Incluso se puede decir que en algunos casos el interés también responde a una "economía de recursos": Ya los estudiantes ni los profesores tienen que movilizarse siempre hasta los centros formativos. Proporcionalmente, el uso de medios electrónicos supone un ahorro en término de trabajos impresos (con su consecuente ahorro en términos ambientales), a la vez que los centros educativos no tienen que prestar siempre sus instalaciones, lo cual implica un ahorro que se puede traducir en términos económicos, etcétera.

Ahora bien, un tema de preocupación que suscitan los medios virtuales es sobre la autoría de los trabajos, en especial de aquellos que implican resolución de tareas de alta complejidad en periodos cortos de tiempo. Se debe pensar por un momento en aquellas tareas que incluyen resolución de problemas matemáticos, físicos o 
químicos... Se tiende a pensar que los alumnos pueden llegar a utilizar a terceros o a los medios que ofrece internet para resolverlos y de esta manera "engañar" o "engañarse" al respecto de su propio rendimiento.

La respuesta más típica que se da es que debe haber un equilibrio entre las tareas 0 evaluaciones que son encargadas por estos medios versus las tareas y evaluaciones realizadas bajo una vigilancia directa de los profesores y en los espacios de clase. Si bien es cierto, esta respuesta no es enteramente satisfactoria, es precisamente una de las condiciones implícitas en cualquier proceso formativo virtual, por lo que es difícil prescindir de ella.

De este modo, ¿qué pasa cuando los procesos de formación son 100\% virtuales? Si se toma por ejemplo, aquellas universidades con programas de estudio que son completamente virtuales entonces la pregunta es totalmente juiciosa. Muchos afirman que esta manera de formarse es igual de válida que la manera tradicional de hacerlo, pero existen serios cuestionamientos que hacen dudar de que así lo sea.

Precisamente, otro de estos cuestionamientos es que la interacción profesor-alumno es totalmente indispensable para que los procesos formativos puedan llegar a ser efectivos y que sin la misma la calidad de la educación es bastante inferior. Esto porque hay muchas situaciones que se producen espontáneamente durante las clases que escapan a la virtualidad, y que son tan o más importantes que los contenidos mismos de los cursos, pues es en esa interacción que surgen las dudas que movilizan el conocimiento.

Esta interacción presencial entre el profesor y pupilo es de suma importancia, sobre todo cuando son cursos en los cuales el profesor necesita realizar una especie de diagnóstico para conocer el grado de aprendizaje y entendimiento de sus estudiantes sobre la materia que imparte. Esta posibilidad se pierde por completo en las clases virtuales, en su mayoría grabadas para que el interesado las pueda ver en el horario de 
su preferencia. Y en el caso que surgiera dudas sobre la materia, solo le quedará la opción de enviar un mensaje o correo al profesor para poder aclarar las incongruencias, y esperar a que esa persona responda en su tiempo o en un escenario peor, no obtener respuesta del todo.

Los partidarios de este tipo de formación dirán que si bien cierto esas interacciones son sumamente importantes, las mismas son reemplazadas por los medios que ofrece internet, como videoconferencias, video foros, correos, tareas, ensayos y demás. Claramente no es una cuestión que se resuelva fácilmente, pero se puede ser del criterio de que los procesos formativos no deberían ser $100 \%$ virtuales, puesto que sí hay algo de ese poder movilizador en la interacción en tiempo real que se pierde absolutamente con la virtualidad.

Otro problema típico que supone la virtualidad en los procesos de formación es la sobreabundancia de material en línea al que podemos tener acceso. Así, aunque mencionamos la posibilidad de acceder a miles de libros y fuentes bibliográficas en línea es necesario tener el conocimiento respectivo para lograr hacer una búsqueda efectiva que nos permita encontrar exactamente lo que buscamos y no naufragar en la web. No se gana algo con que exista esta abundancia de medios si no se tiene el conocimiento que permita saber cómo y en donde buscar, ni tampoco con buscar material relacionado con un interés particular, pero pudiera ser que el nivel 0 profundidad de ese material no sea acorde con el nivel que se posea como estudiante; y en este punto las instituciones educativas y en especial la secundaria y las universidades deberían cumplir un papel formativo importante.

Aunado a lo anterior, está el tema de la validez de las fuentes. Porque de todos es bien sabido que cualquier persona puede subir contenidos a la red, sin que estos tengan la validez necesaria para ser tomadas en cuenta. Para esto los estándares internacionales han establecido las reglas que permiten separar las fuentes válidas de las que no lo son, y las normas ISSN e ISBN son un muy buen ejemplo. 
Se vuelve imperativo considerar lo dicho por (Silva Quiroz, 2014) citando a Marc Prensky:

La tendencia actual y futura indica que la evolución de la tecnología en la sala de clases será personalizada a través de dispositivos portátiles, inalámbricos y permanentemente conectados, no obstante, estos estudiantes suelen tener problemas al enfrentarse al mundo educativo, dado que sus docentes, inmigrantes digitales, Prensky, (2001), siguen ofreciendo los mismos entornos tradicionales de aprendizaje. (p.3)

Considerando todo lo dicho hasta acá y para ampliar sobre los aspectos que ejerce la virtualidad en los procesos formativos, nos referiremos ahora a su papel en el ámbito laboral. Sobre esto (Calvo Verdú, 2005) se refiere a las diferentes ventajas de integrar nuevas tecnologías en la formación, además de cómo se debe asumir este reto:

La integración de las nuevas tecnologías como medios didácticos en la formación que facilitan los procesos de enseñanza y aprendizaje, supone el primer nivel introductorio porque, en realidad, los objetivos son mucho más amplios: la introducción de las mismas en la educación en general. Por tanto, el formador no debe quedarse en la mera introducción en el uso de las nuevas tecnologías en la metodología didáctica, sino que debe plantearse seriamente el cómo se produce el aprendizaje con los elementos multimedia, qué posibles relaciones comunicativas se pueden crear y qué nuevos roles asumen el formador y los alumnos en el aprendizaje. A todo ello se suma el abordar todos los aspectos novedosos que se introducen en el uso de las nuevas tecnologías desde una perspectiva crítica y creadora, llegando con ello a un segundo nivel de profundización en las relaciones entre las Nuevas Tecnologías y la Formación (p.200)

Asimismo, este autor nos plantea como ventajas de la implementación tecnológica la innovación en la práctica docente, lo cual quiere decir que los medios informáticos permiten adaptarse al ritmo diferente de aprendizaje de los aprendientes gracias a su 
flexibilidad y adaptabilidad. Como otra ventaja, nos indica que mejora la organización y la gestión formativa, esto debido a que se consigue una optimización sustancial de las tareas administrativas y formativas.

Sin embargo, a pesar de las muchas ventajas de implementar la virtualidad en las empresas, como cualquier herramienta de trabajo, se encontrará con el factor negativo, es decir, que este sistema puede contener puntos débiles o desventajas tanto para la el patrón como para el colaborador, entre algunas de ellas se pueden citar las siguientes:

La empresa que por primera vez requiere implementar la virtualidad, debe contar con el presupuesto adecuado para cubrir todos los gastos que esto implica. Esto se traduce en los gastos por concepto de compra de equipos, ya que no en cualquiera de estos se puede instalar los programas adecuados. Además de esto, es requisito contar con el personal idóneo o con los conocimientos necesarios en estos sistemas de información, para que los mismos se encarguen de realizar las funciones respectivas cuando se desea programar alguna capacitación virtual, en caso de no tenerlos, la empresa debe gestionar su contratación. El contar con este personal es sumamente importante para que estos sistemas sea eficaces en una empresa, como es de conocimiento, todo equipo necesita mantenimiento constante así como la actualización de su software. Por lo tanto, una empresa debe financiar el presupuesto no solo para la instalación de estos programas virtuales, sino que también deben tener el noción, que los mismos van a demandar una serie gastos durante el tiempo que la empresa estime conveniente tenerlos, de lo contrario la empresa se vería en la necesidad, ya sea de reducir sus procesos formativos llevados a cabo de la forma tradicional, o bien omitirlos.

$>$ Otro punto débil que puede conllevar la virtualidad, va en función de sus empleados y se ve reflejado en la resistencia al cambio que los mismos experimentan con respecto a esta herramienta. Esta resistencia se suele 
presentar la mayor parte de las veces en aquellos empleados que llevan años trabajando en la empresa, y se acostumbraron a asistir a capacitaciones u otras maneras de formación tradicional, es decir, en forma presencial- magistral, también se suele dar en trabajadores con edades avanzadas. Por lo cual, es responsabilidad de la empresa, motivarlos para hacer ese cambio, de manera que los mismos se adapten a las nuevas tecnologías. (Calvo Verdú, 2005).

Como algunos autores lo han señalado (Imbernón Muñoz, Silva García, \& Guzmán Valenzuela, 2011), los procesos formativos que se apoyan en la virtualidad plantean la necesidad de desarrollar y potencializar competencias específicas que tal vez hasta ahora no habían sido tan determinantes. Esa es una de las tareas pendientes de considerar por las personas que deseen abocarse a este tema de estudio, que representa todo un reto para el futuro.

Otra desventaja que podría enmarcarse en esta línea, concerniente al capital humano, sucede cuando algunos colaboradores que por diferentes razones se hallen desmotivados o bien indiferentes a la implementación tecnológica, simplemente se unan a las videoconferencias, pero realmente no presten mucha atención o bien dejen "correr el video o programa" mientras se abocan a realizar otras tareas. Esto es una práctica generalizada en muchas empresas, y más aún en situaciones donde el empleado labora desde la casa ciertos días a la semana y no pone interés en estas herramientas didácticas que facilita la virtualidad.

\section{Conclusión}

La educación ha sido y será siempre una tarea bastante compleja. Desde el mismo momento del nacimiento, nos envuelve y nos exponemos a muchos y muy variados procesos de aprendizaje. La educación es una manera destacada y sumamente 
depurada que tenemos intrínsecamente para identificar, transmitir e incluso para prevalecer como seres especie humana.

Se puede decir que la virtualidad constituye una herramienta muy valiosa para toda empresa exitosa que cuente con una buena estrategia, y como parte de esta estrategia se encuentre la innovación, la cual va ligada al proceso de adaptación a los cambios tecnológicos que se generan día con día. Como se explicó anteriormente, en el ámbito laboral la virtualidad no se utiliza solo para agilizar procesos operativos 0 administrativos del comercio, sino que también ejerce un rol primordial en la preparación de los colaboradores, es decir, esta llega satisfacer una serie de necesidades de ambas partes (empresa-empleado).

En el ámbito educativo, la implementación de nuevas tecnologías será una herramienta eficaz en la investigación de nuevos materiales didácticos, y como facilitador de las tareas diarias tanto del profesor como del alumno. Todo esto con el pequeño inconveniente que se pierde el contacto visual entre profesor-estudiante y las posibles dudas que podrían ser aclaradas inmediatamente solo si fuera una clase en vivo y presencial.

Algunos han visto e incluso hasta vivido la invasión de la tecnología en la educación hasta como un gran peligro, más que una amenaza para lo que habían aprendido que era el supuesto correcto proceso educativo. La casi pérdida de la relación entre facilitador $y$ aprendiente, en tanto para otros ha sido la oportunidad perfecta para innovar en la clase y poder llegar a aquellos para quienes el proceso formativo era solo un sueño ya sea por sus recursos económicos limitados o la imposibilidad de trasladarse a los centros de enseñanza.

La virtualidad ofrece infinidad de posibilidades para crear entornos nuevos de relación, interacciones y sinergias que como tales, deben de ser tratados de una forma no tradicional para potenciar, extraer y aprovechar lo mejor de todos los involucrados en 
los procesos de aprendizaje. La riqueza y las grandes posibilidades que adquieren estos nuevos entornos, aún no tan explorados, es inmensa y su poderío se combina con la tecnología y todo nuevo conocimiento incluso por venir. Por risible que parezca, lo único constante y permanente en la vida es el cambio en sí mismo, no se debe estar cerrado ante las nuevas tendencias y sobre todo cuando parecen traer un sello de innovación y mejora.

Por último, es importante señalar que conforme las formas existentes de entender y de renovar las maneras como se enseña, tanto en contextos educativos como laborales, el uso de la tecnología es y será cada vez más fundamental. El tema estará entonces en poder entender cómo implementarla de modo tal que sea un elemento posibilitador y motor del cambio, al tiempo que redefinimos los papeles del profesor y estudiante en el aprendizaje.

\section{Referencias}

Barberà, E. \& Badia, A. (Noviembre, 2005). El uso educativo de las aulas virtuales emergentes en la educación superior. Revista de Universidad y Sociedad del Conocimiento, II(2), 1-12.

Calvo, M. (2005). Formador ocupacional. Formador de formadores. Formación profesional ocupacional. Temario, test y casos prácticos (Primera ed.). Sevilla, España: Editorial Mad, S.L.

Imbernón, F., Silva García, P. \& Guzmán, C. (2011). Competencias en los procesos de enseñanza-aprendizaje virtual y semipresencial. Comunicar: Revista científica de comunicación y educación, XVIII(36), 107-114.

Silva, J. E. (2014). La virtualidad una oportunidad para innovar en educación: Un modelo para el diseño de entornos virtuales de aprendizaje. Revista Didasc@lia: $D \& E, V(1), 1-22$. 